Todua Ana, Chumburidze Rusudan

Ivane Javakhishvili Tbilisi State University

\title{
Integrated Learning of the Georgian Language and Civic Education within the " $1+4$ " program
}

\begin{abstract}
Integration of minorities and civil involvement are some of the most important components of a country's democratic development. The changes made in the education policy of Georgia, that were executed on November 19, 2009 were aimed to strengthen integration of ethnic minorities, their access to education and civic engagement. It has been 5 years already since these changes led to establishing a successful preferential policy for ethnic Armenian and Azerbaijani high school graduates entering higher education institutions.

During this period, several educational-methodical guidelines have been developed and different methods of learning have been introduced within the framework of the Georgian Language Education Program. However, the necessity of the methodological improvement of the Georgian language learning and the introduction of modern and systematic approaches is still essential. In the course of this study the need for pragmatic approach to language teaching became evident, where emphasis will be made on the development of communicative competence. This approach of language learning implies diversity and general change of topics and text types. Within this method, the student is not only a passive participant, but also a co-creator of knowledge. An Integration method, together with language learning, will increase the students' competence in different directions, most importantly in civic education, as well as will furnish them with presentation, communication, teamwork and free speech skills, allowing students to be informed, to be able to state their own position and help them to become active citizens. An Integrated Learning of Georgian Language and Civic Education program effectively meets all these criteria.

The efficiency of the language and subject integrated teaching approach in a higher educational institution is proved by the completed project "Earth Calendar", which was financed by the "Open Society Foundation - Georgia". Such a resource had been created, which was adjusted to the integrated teaching approach of the language and subject at the university level.

Within the project, a study and a course of training have been carried out. Also, the textbook "Earth Calendar" for the TSU Preparatory (Prep) Program within the piloting policy has been piloted during the term.

It should be said, that the approach was successful. By the end of the term, the feedback has been obtained, which proved the efficiency of the integrated teaching of a language and Civic Education and the urgency of the topics given in the textbook.
\end{abstract}

Key words: Integration of minorities, language learning, integrated teaching of a language and Civic Education

Integration of minorities and civil involvement are two of the most important components of the country's democratic development. Isolation regarding lack of information is considered as a hindering factor of integration, which is directly linked to the access to education for minorities. 
Integration of ethnic minorities, access to education and civic engagement are aimed to strengthen the changes that took place in education policy, which came into force in November 19, 2009, and is concerned with the higher education institutions presenting ethnic Armenian and Azerbaijani with a more advantageous policy of enrolment.

In Georgian higher education institutions for the past 7 years, students of minority groups have been successfully enrolled in a Georgian language preparatory program, which is often referred to as the "1 + 4" program. Based on the statistical data, from 2010 until now, most students have joined TSU Jano Javakhishvili University, which, of course, indicates the success of the program.

From the beginning of the program up to now, TSU has developed a number of teaching methods within the frames of the educational program of teaching the Georgian language. However, it should also be noted, that the necessity of implementing methodological improvements of the Georgian language teaching and modern, systematic approaches are still relevant. Most of the educational-methodical textbooks are only focused on teaching the Georgian language, which, based on our experiences, because of the specifics of students of this program, are not effective. Moreover, participation in lecture activities is not a panacea for the development of civic consciousness and intercultural sensitivity of these students. In spite of studying the language at a certain level, the representatives of ethnic minorities (with certain exceptions) are less integrated into Georgianspeaking societies from the social-cultural point of view. Taking this into consideration, teaching methodical textbooks on the program " $1+4 "$ are desirable to raise the civic awareness of the students as well. We have conducted our research in this direction, employing the method of in-depth interviews and questionnaires. Within the scope of the study the students anonymously answered the questionnaires created by a sociologist: they assessed the textbooks and were asked to give feedback regarding the needs and desires concerning the academic process, method or approach. Over 300 students were interviewed. The results of the study showed that students were interested in activities such as civic education, information-awareness and entertainment topics. Taking the above mentioned into consideration, we decided to write a book, within the framework of 1 +4 program, based on new guidelines and methods ( such an integrated teaching of the language and civic education) where the emphasis in the teaching process would be on developing communication competences.

This approach of language learning implies the diversity and systemic changes regarding the topics and text types, as a student is not only a passive participant but also a co-creator of knowledge. As a sort of reflection, we decided to set up an approach that would fit into the " $1+4 "$ program specifications. Taking into account the fact that students were less integrated in Georgian society, thus were less active in civic activities, the method of integration of the Georgian language and civic education was selected.

Many academic research has been carried out on the CLIL methodology. In most cases, it is considered to be an effective method and, moreover, nowadays, it is actively used in different countries in the process of language teaching all around the world.

A traditional method was adjusted to " $1+4 "$ program and we made an attempt to find a necessary direction for students - civic education. Along with the aspect of language teaching, topics were selected to focus on increasing civic consciousness of students.

Why civic education? Raising civic awareness in young people starts at a school age and involves the development of important skills such as assessment and correction of their own perceptions and behaviors, understanding and protection of the rights and obligations of self and others, involvement in civic activities and volunteering, setting goals; generating ideas, initiations and ways to implement them, caring for life in every day and emergency situations, following a healthy lifestyle, etc. The list as we see is quite large and essential for life.

Our objectives are to: 1 . Promote social and cultural integration into a Georgian-language environment of Armenian and Azeri students of national minorities; 2. Expand their knowledge, introduction of complex activities in lessons, along with language teaching, raise students' competencies in different directions (for example, reasoning, convincing, presentation, creative skills, etc.); 3 . Adjusting the calendar event format onto 
E ISSN 1512-3146 (online) ISSN 1987-9601 (print)
International Journal

of Multilingual Education

the study process. Having this in mind, the project " the Earth Calendar" was written and is already funded and implemented by "Open Society Georgia" Foundation. Within the framework of the project, a methodical guide has been developed which has already been successfully implemented in the pilot format for the $1+4$ program. The book, as we have already mentioned, is aimed at raising civic awareness and the topics are selected in this regard. Together with the use of the integration method, a new approach has been followed, which we believe is a novelty for the $1+4$ program and makes it more effective and interesting. The topics selected in the book coincide with the dates (e.g., AIDS Day - 1 December, Earth Day - 22 April, Autism Day - April 2, Tree Planting Day - October 22, International Day for Volunteers - December 5, International Science Day November 11, Earth Hour - March 28, etc.), which make the topics not only interesting, but also relevant for the students. This gives them the opportunity to be informed and to express their opinion and to be involved in a variety of fun activities.

I think the adjustment of the calendar format is a methodical tool for the lecturer. Besides the available material and educational resources, it is also possible to plan classroom work in a manner that reflects the reality, and develops communication skills of the student.

In addition, the above mentioned activities will facilitate the process of studying the language. As well as this, it will be a kind of cognitive process in different directions, such as civic education, gender issues, cultural diversity, tolerance, advocacy, education...

It is noteworthy that by studying important dates and participating in these activities, students are presented with the opportunity to expand the worldview, reflect on their own culture in the context of other cultures; become yet more integrated into the Georgian culture and at the same time perceive themselves as a world citizen.

The criteria below were used to create the " $1+4 "$ program employed to prepare the material to work with the students of the program.

The study material was selected according to the following principles: 1 . Consideration of the calendar format approach; 2. Interesting and relevant issues regarding the age of the students (30 topics); 3 . Focusing on topical vocabulary.

Criteria for selection of topics were as follows: 1. Awareness - raising (civil, cultural and social integration); 2. Functional - oriented towards raising conscious awareness (in real social situations) 3. Something unusual, original, different, varied, fun. Taking everything into consideration, the topics we have chosen may be grouped as follows:

In the block, which combines the importance of ecology and healthy lifestyle; combined topics: hiking, flower and tree planting day, wildlife day, earth day, world without tobacco;

Raising civil awareness and diversity: Cultural Diversity Day, Native Language Day, Flag Day, Down Syndrome Day, Elderly Day, Day of Kindness;

Topics concerning human rights and education: Mutual Respect Day, Greeting Day, Free Press Day, Compliment Day, Literature Day, Museum Day, Science Day, Firefighter Day;

Information-related topics include: Tea Day, New Year, Hug Day, Unusual Hobby Day, Board Games Day, Clock Ticking Day, Bread Day, Good Neighbor Day.

All topics are made up of methodically worked out structures.

In order to illustrate how the above mentioned methodical approaches are used during the lecture, one topic from the textbook is given as an example.

Mutual Respect Day, which is celebrated on September 18, is developed using a variety of activities, the number of which reaches 24 and includes various types of tasks. The processing of the topic begins with the introduction of the content, the keywords and list of key issues that will be processed during the lecture; 
- 'Before' reading activity: brainstorming that aims to elicit students' opinions on the issue, and to spark interest in them (e.g., what does the word respect mean? Mutual respect; name several people you respect; explain how these people deserve respect);

- Work on vocabulary and dictionaries (e.g., searching for words in the definition / synonyms dictionary (e.g., finding words that are synonymous with the word respect);

- Creation of an associative and concept map;

- Reading and processing the first base text (with the accompanying tasks that are intended for understanding and analyzing the text: questions and answers, open and multiple choice tests, finishing sentences, etc.);

- Processing of audiovisual resources (for example, conveying content, discussing animations: what is respect and appreciation, how is the essence of respect depicted in the animation? How convincing is it? What would you change in the animation?);

- Rôle Play / Dialogues: To select the desired option from the list of suggested options and present it together with the partner (e.g. answer the question politely; behave politely in public places - in the shop - queue, in the street if you accidentally bump into someone...)

- Coming up with a short story based on the suggested photos;

- Read and process the second text (with the accompanying tasks that are intended for understanding and analyzing the text: question-answer, open and multiple choice tests, inserting relevant keywords in the sentences, justifying the choices);

- Reading and processing of the third text (question-answer, open and multiple choice tests, inserting relevant keywords in the sentences, giving titles to the text/letter, discussion, substantiating opinions, making sentences using key words; describing (real / imaginary) a person, who has earned respect, individually or together with a partner);

- Reading and processing of the fourth text (with the accompanying tasks intended for understanding and analyzing the text: question- answer, true / false, open and multiple choice tests, inserting relevant / synonymous words in the sentences, making up sentences using key words, etc.);

- Processing of audiovisual resources (video, "Good / Bad Team"- talking about the content, , discussing, summarizing content);

- Generating discussion about the topic (with the help of the support questions for the discussion: how should we behave, what should we do and not do in the learning environment, how to behave equally politely towards everyone? What are the differences between the good and bad teams? What is a must to succeed in team work?);

- Ideas for assignments that can be used in the classroom as well as homework (find / remember the definition of "respect"), describe how you did and did not express respect to others; make a Respectable People list and explain the reason why they were included in the list; Interview - think of some important questions you would ask these respectable people at the interview; individually or with a partner, make a poster about Mutual Respect Day; think of (look up in the dictionary) five words that can be used as antonyms of the word "respect"; Imagine how the world would change if more people expressed respect for each other; find five photos in which, in your opinion, is reflected the notion of "respect", etc.

As can be seen, activities are quite diverse and interesting. Texts presented belong to different types both printed and multimedia: information-cognitive, scientific, letters, etc. There are a lot of texts, but that does not mean that employing all of them is compulsory. The lecturer will select an appropriate text and activities according to students' interest and study objectives.

In order to test the method and confirm its effectiveness, a research and training cycle was conducted within the project, and a supporting textbook for the TSU preparatory program: "Earth Calendar" was tested 
in the pilot mode during the semester. It can be said that the approach was successful. The feedback at the end of the semester confirmed the effectiveness of integration of the language and civic education and also, the importance of the topics presented in the manual.

Consequently, we think the introduction of the method of CLIL into the university level will be an effective tool for the language use. This approach will help students of the " $1+4$ " program to increase their language and civic awareness.

\section{References}

CLIL - Content and Language Integrated Learning (Do Koyle, Philip Hood and David Marcy) 2016.

Submission for the Universal Periodic Review on Georgia on the Rights of National Minorities in Georgia, Prepared by: Public Movement "Multinational Georgia, 11.07.2010.

Textbook "the Earth Calendar" in the Georgian Language Preparatory Program. Tbilisi, 2017, p. 21.

The United Nations Association of Georgia "Strengthening National Integration in Georgia" - Civil Integration Research, 2014. 